\title{
An immunohistochemical study of the diagnostic value of TREM-1 as marker for fatal sepsis cases
}

\author{
A. Maiese, G. Bolino, A. Mastracchio, P. Frati \& V. Fineschi
}

To cite this article: A. Maiese, G. Bolino, A. Mastracchio, P. Frati \& V. Fineschi (2018): An immunohistochemical study of the diagnostic value of TREM-1 as marker for fatal sepsis cases, Biotechnic \& Histochemistry, DOI: 10.1080/10520295.2018.1535138

To link to this article: https://doi.org/10.1080/10520295.2018.1535138

曲 Published online: 02 Nov 2018.

Submit your article to this journal $\pi$

Џll Article views: 60

View Crossmark data $₫$ 


\title{
An immunohistochemical study of the diagnostic value of TREM-1 as marker for fatal sepsis cases
}

\author{
A. Maiese $\mathrm{a}^{\mathrm{a}, \mathrm{b}}, \mathrm{G}$. Bolino ${ }^{\mathrm{a}}$, A. Mastracchioc, P. Fratia ${ }^{\mathrm{a}, \mathrm{b}}$, and V. Fineschi ${ }^{\mathrm{a}, \mathrm{b}}$ \\ aDepartment of Anatomical, Histological, Forensic and Orthopaedic Sciences, Sapienza University of Rome, Rome, Italy; 'bIRCCS Neuromed, \\ Pozzilli, Italy; 'Medical UOC Pathology Local Health Agency Rome/6, University of Rome "Tor Vergata", Rome, Italy
}

\section{ABSTRACT}

Triggering receptor expressed on myeloid cells-1 (TREM-1) is produced and up-regulated by exposure of myeloid cells to lipopolysaccharides or other components of either bacterial or fungal origin, which causes it to be strongly expressed on phagocytes that accumulate in inflamed areas. Because TREM-1 participates in septic shock and in amplifying the inflammatory response to bacterial and fungal infections, we believe it could be an immunohistochemical marker for postmortem diagnosis of sepsis. We tested the anti-TREM- 1 antibody in 28 cases of death by septic shock and divided them into two groups. The diagnosis was made according to the criteria of the Surviving Sepsis Campaign. In all cases, blood cultures were positive. The first group was comprised subjects that presented high ante-mortem serum procalcitonin and the soluble form of TREM-1 (s-TREM-1) values. The second group comprised subjects in which s-TREM-1 was not measured ante-mortem. We used samples of brain, heart, lung, liver and kidney for each case to test the anti-TREM-1 antibody. A semiquantitative evaluation of the immunohistochemical findings was made. In lung samples, we found immunostaining in the cells of the monocyte line in 24 of 28 cases, which suggests that TREM-1 is produced principally by cells of the monocyte line. In liver tissue, we found low TREM-staining in the hepatocyte cytoplasm, duct epithelium, the portalbiliary space and blood vessel. In kidney tissue samples, we found the TREM-1 antibody immunostaining in glomeruli and renal tubules. We also found TREM-1 staining in the lumen of blood vessels. Immunohistochemical staining using the anti-TREM-1 antibody can be useful for postmortem diagnosis of sepsis.

\section{KEYWORDS}

Immunohistochemistry; marker; postmortem

diagnosis; sepsis; septic myocardial dysfunction; septic shock; triggering receptor expressed on myeloid cells-1
Triggering receptor expressed on myeloid cells-1 (TREM-1) belongs to the immunoglobulin superfamily (Barraud and Gibot 2011). It is expressed on neutrophils and mature monocytes, and the genes that code it are grouped on human chromosome 6. TREM-1 is expressed also on nonmyeloid cells, such as epithelial or endothelial cells (Bouchon et al. 2000). TREM-1 is produced and up-regulated by exposure of myeloid cells to lipopolysaccharides or other microbial components of either bacterial or fungal origin, which causes it to be strongly expressed on phagocytes that accumulate in inflamed areas (Bleharski et al. 2003; Yuan et al. 2012).

TREM-1 has an alternatively spliced variant that lacks the transmembrane region, which results in secretion of the receptor in a soluble form (s-TREM-1) (Weiss et al. 2017). s-TREM-1 is a $17-\mathrm{kDa}$ fragment that is detectable in body fluids such as blood, broncho-alveolar lavage and cerebrospinal fluid. This molecule can be dosed in biological fluids (Brenner et al. 2016). Plasma levels of
s-TREM-1 $>60 \mathrm{ng} / \mathrm{ml}$ seem to be more accurate than other clinical or laboratory tests for identifying patients with an ongoing infection (Giamarellos-Bourboulis et al. 2006). s-TREM-1 is released by activated phagocytes and can be found in plasma, pleural fluid, cerebrospinal liquor and urine (Arízaga-Ballesterosa et al. 2015; Latour-Pérez et al. 2010; Wu et al. 2012).

Combined with other markers, s-TREM-1 appears to be valuable for differential diagnosis of a noninfective sepsis with systemic inflammatory response syndrome (SIRS) (Bouchon et al. 2001; Oku et al. 2013). In the forensic field, reliable diagnosis of sepsis may be even more difficult owing to unavailable medical records at autopsy, nonspecific autopsy and histological findings including myocardial ischemia, pulmonary edema, hypoxic liver damage, mesenteric ischemia, gastrointestinal hemorrhage, spleen infarction, kidney ischemia and brain edema that may have either an infectious or non-infectious origin.

CONTACT V. Fineschi vfinesc@tin.it Department of Anatomical, Histological, Forensic and Orthopaedic Sciences, Sapienza University of Rome, Viale Regina Elena 336, Rome 00185, Italy

(c) 2018 The Biological Stain Commission 
Because of the crucial role that TREM-1 plays in increasing septic shock and amplifying the inflammatory response to bacterial and fungal infections, we suspect that it could be a useful immunohistochemical marker for postmortem diagnosis of sepsis.

\section{Material and methods}

Our data are covered by the general authorization to process personal data for scientific research purposes granted by the Italian Data Protection Authority (1 March 2012 as published in Italy's Official Journal no. 72 dated March 26 2012), because the data do not include significant personal information concerning the subjects. Our study does not involve the application of experimental protocols; therefore, it does not require approval by institutional and/or licensing committees. In all cases, local prosecutors opened an investigation and ordered that an autopsy be performed to clarify the exact cause of death. We selected seven women and 21 men, mean age 58.2 years, who died from septic shock with an ante-mortem diagnosis of sepsis. The diagnosis was made according to the criteria of the Surviving Sepsis Campaign (Singer et al. 2016). Blood cultures were positive in all cases. We subdivided the cases into two groups. The first group comprised 14 subjects that presented high ante-mortem serum procalcitonin and s-TREM-1 values. The diagnostic evaluation of s-TREM-1 was performed using receiver operating characteristic (ROC) analysis. Areas under the curve (AUCs) were calculated. The second group comprised 14 subjects in which s-TREM-1 was not measured antemortem. Data concerning the subjects are given in Table 1.

We chose non-infective cases as a control group of adults ( $\mathrm{n}=14$; six women, eight men, mean age 49.4 years) with no clinical history or macroscopic or microscopic signs of infection whose deaths were characterized by suddenness. We tested the anti-TREM-1 antibody on subject that died from opioid overdose $(\mathrm{n}=5)$, hanging $(\mathrm{n}=4)$, car accident $(\mathrm{n}=4)$ and sudden cardiac death $(\mathrm{n}=1)$. The postmortem interval was $\leq 36 \mathrm{~h}$ in each case. We used five samples, brain, heart, lung, liver and kidney for each case.

Samples, $8 \mathrm{~cm}^{2}$, of each organ from each case were fixed in $10 \%$ buffered formalin, then washed with phosphate-buffered saline (PBS) and subsequent dehydration was carried out using a graded alcohol series. After dehydration, samples were cleared in xylene, and embedded in paraffin. Sections were cut at $4 \mu \mathrm{m}$, mounted on slides and covered with 3-amminopropyltriethoxysilane (Fluka, Buchs, Switzerland). A routine microscopic histopathological study was performed
Table 1. Ante-mortem serum and main autopsy and histology findings.

\begin{tabular}{|c|c|c|c|c|}
\hline Case & $\begin{array}{c}\text { PCT } \\
\text { ante- } \\
\text { mortem }\end{array}$ & $\begin{array}{c}\text { s-TREM- } \\
1 \\
\text { ante- } \\
\text { mortem }\end{array}$ & $\begin{array}{l}\text { Ante-mortem } \\
\text { microbiology in blood }\end{array}$ & $\begin{array}{l}\text { Main autopsy and } \\
\text { histology findings }\end{array}$ \\
\hline 1 & 29.8 & 120.0 & $\begin{array}{l}\text { Acinetobacter } \\
\text { baumannii }+ \text { S. aureus }\end{array}$ & Pneumonia \\
\hline 2 & 17.34 & 80.4 & E. coli & Peritonitis \\
\hline 3 & 16.22 & 64.15 & K. pneumoniae & Pneumonia \\
\hline 4 & 37.04 & 100.74 & S. aureus & $\begin{array}{l}\text { Necrotizing fasciitis } \\
+ \text { pneumonia }\end{array}$ \\
\hline 5 & 25.15 & 75.20 & E. faecalis + E. coli & Peritonitis \\
\hline 6 & 20.15 & 88.50 & $\begin{array}{l}\text { St.epidermidis + } \\
\text { S. aureus }\end{array}$ & $\begin{array}{l}\text { Pneumonia-hip } \\
\text { prosthesis infection }\end{array}$ \\
\hline 7 & 33.08 & 93.13 & E. faecalis + E. coli & $\begin{array}{l}\text { Abdominal and } \\
\text { pelvic infection }\end{array}$ \\
\hline 8 & 28.09 & 97.90 & $\begin{array}{l}\text { E. coli } \\
+ \text { P. aeruginosa }\end{array}$ & $\begin{array}{l}\text { Pneumonia } \\
+ \text { urinary infection }\end{array}$ \\
\hline 9 & 18.64 & 68.75 & $\begin{array}{l}\text { Acinetobacter } \\
\text { baumannii }\end{array}$ & Pneumonia \\
\hline 10 & 30.44 & 98.00 & K. pneumoniae & Pneumonia \\
\hline 11 & 16.44 & 66.88 & S. aureus & Pneumonia \\
\hline 12 & 32.42 & 90.26 & P. aeruginosa & Pneumonia \\
\hline 13 & 18.64 & 86.68 & E. coli & Peritonitis \\
\hline 14 & 34.76 & 98.24 & K. pneumoniae & Pneumonia \\
\hline 15 & & & K. pneumoniae & Pneumonia \\
\hline 16 & & & P. aeruginosa & Pneumonia \\
\hline 17 & & & E. coli $+K$. pneumoniae & $\begin{array}{l}\text { Abdominal and } \\
\text { pelvic infection }\end{array}$ \\
\hline 18 & & & P. aeruginosa & Necrotizing fasciitis \\
\hline 19 & & & $\begin{array}{l}\text { L. monocytogenes } \\
+K \text {. Pneumoniae }\end{array}$ & Pneumonia \\
\hline 20 & & & $\begin{array}{l}\text { Stenotrophomonas } \\
\text { maltophilia } \\
+P . \text { aeruginosa }\end{array}$ & Pneumonia \\
\hline 21 & & & $P$. aeruginosa & Pneumonia \\
\hline 22 & & & $\begin{array}{l}\text { P. aeruginosa } \\
+S \text {. Aureus }\end{array}$ & $\begin{array}{l}\text { Pneumonia } \\
+ \text { peritonitis }\end{array}$ \\
\hline 23 & & & Acinetobacter baumanii & Pneumonia \\
\hline 24 & & & E. Coli & Peritonitis \\
\hline 25 & & & Acinetobacter baumanii & Pneumonia \\
\hline 26 & & & K. pneumoniae & Pneumonia \\
\hline 27 & & & L. Monocytogenes & Pneumonia \\
\hline 28 & & & E. Coli & Peritonitis \\
\hline
\end{tabular}

after hematoxylin and eosin ( $\mathrm{H} \& \mathrm{E})$ staining (Fischer et al. 2008).

To test the anti-TREM-1 antibody (clone L5-B8.2A12.3A12; Novusbio, Abingdon, UK), we used a biopsy sample of stomach wall from a living patient with chronic gastritis caused by a Helicobacter pylori infection. The biopsy sample was fixed in $10 \%$ buffered formalin, then washed with PBS and subsequent dehydration was carried out using a graded alcohol series. After dehydration, samples were cleared in xylene and embedded in paraffin. Sections were cut at $4 \mu \mathrm{m}$, mounted on slides and covered with 3-amminopropyltriethoxysilane (Fluka, Buchs, Switzerland). The sections were re-hydrated through graded alcohols and incubated for $20 \mathrm{~min}$ in methanol containing $10 \%$ $\mathrm{H}_{2} \mathrm{O}_{2}$ to block endogenous peroxidases. The sections were pretreated with citrate buffer $(10 \mathrm{mM}$ sodium citrate, $\mathrm{pH}$ 6.0), incubated with the anti-TREM-1 antibody (clone L5-B8.2A12.3A12; Novusbio, Abingdon, UK) diluted 1:500, then treated with BrightVision ${ }^{\circ}$ 
PolyHRP-anti rabbit (Vector Labs, Peterborough, UK) and developed using $1 \% \mathrm{H}_{2} \mathrm{O}_{2}$ and DAB (SigmaAldrich, Darmstadt, Germany) in $0.05 \mathrm{M}$ Tris- $\mathrm{HCl}$ (pH 7.9) (Yuan et al. 2014). The anti-TREM-1 exhibited staining in the stomach epithelium and glands, which served as a positive control. All postmortem samples from brain, heart, lung, liver and kidney, were immunostained using the method described above for the stomach wall biopsy.

For quantitative analysis, in each immunohistochemical section we made 20 observations in different fields/slide. The TREM-1 stained cells were counted at $40 \mathrm{x}$ using a light microscope coupled to a high resolution color video camera. The number of stained cells was divided by the total number of cells and multiplied $\mathrm{x} 100$. The immunohistochemical positivity score was defined as: 0 , nuclear and/or cytoplasm staining absent; 1 , nuclear and/or cytoplasmic staining, 25\%; 2, nuclear and/or cytoplasmic staining, 50\%; 3, nuclear and/or cytoplasmic staining, 75\%; 4, nuclear and/or cytoplasm staining, $100 \%$. We also quantified immunohistochemical staining in blood vessel as: 0 , absent; 1 , low, 25\%; 2 , sufficient, $50 \%$; 3 , good, $75 \%$; 4 , considerable, $100 \%$. A subjective scoring system for the staining intensity of cells was defined. Five degrees of intensity were defined as: 0 , absent; 1 , low, $25 \%$; 2 , sufficient, $50 \%$; 3 , good, $75 \%$; 4 , considerable, $100 \%$ ). The most intense coloration, grade 5, was that of the stomach wall sample with chronic gastritis that was used as the positive control. In cases of heterogeneous intensity, we considered the prevailing intensity. All evaluations were performed in a double blind manner. In cases of divergent scoring, a third observer decided the final score.

\section{Results}

Our findings are summarized in Tables 2 and 3. We found blood vessel immunostaining in kidney tissue samples in 25 cases (Figure 1a). In lung, we found clear staining in lumen of the blood vessels and cytoplasm of myelomonocytes in 24 of 28 cases (Figure 1b). In myocardial samples, we found clear immunostaining in blood vessels and no staining of the cytoplasm or nucleus of myocardial cells (Figure 1c). No anti-TREM-1 staining was found in any brain samples or nuclei of nerve cells; we found blood vessel staining in all cases (Figure 1d). In lung samples we found immunostaining in the cells of the monocyte line in 24 of 28 cases, which suggests that TREM-1 might be produced principally by cells of the monocyte line (Figure 2a). We found little staining in hepatocytes of the liver in 21 of 28 cases (Figure 2b). In 14 cases, immunostaining appeared in the duct epithelium or in the portal-biliary space, and in 18 cases we found immunostaining in the blood vessels. In 25 of 28 kidney tissue samples, we found immunostaining in the glomeruli (Figure 3a), in 20 cases we found staining in the renal tubules and in 25 cases we found staining of blood vessels (Figure 3b).

Our findings for the two groups were similar, but the first group showed a greater immunohistochemical staining and intensity. The anti-TREM-1 antibody exhibited no reaction in organs or blood vessels of cadavers who died from non-infective causes (control group).

Finally, we detected greater TREM1 staining in the lumen of the blood vessels of first group with documented ante-mortem s-TREM-1 concentrations (Figure $4 a-d)$.

\section{Discussion}

We used an experimental model and immunohistochemistry to conduct an investigation of postmortem TREM-1 expression. Various immunohistochemical markers have been used to investigate cases of sepsis, especially with regard to the lung, including markers for E-selectin, very late antigen 4 (VLA-4), intracellular adhesion molecule 1 (ICAM-1) and lactoferrin (Müller et al. 2008; Herwig et al. 2013; Galassi et al. 2018).

Tumor necrosis factor alpha (TNF- $\alpha$ ), interleukin-1 beta (IL-1 $\beta$ ) and interleukin-6 (IL-6) are found intermittently or briefly during an inflammatory response and during sepsis (Carsin et al. 1997). We reported earlier that anti-procalcitonin antibody can be a valuable tool for postmortem diagnosis of sepsis (Maiese et al. 2017). Nevertheless, definitive techniques that can be used to make a postmortem diagnosis of sepsis are lacking. Macroscopic and microscopic pathological findings are nonspecific and may be inadequate to render a definitive diagnosis for forensic purposes when clinical documentation is lacking (Tsokos 2007).

Gibot et al. (2004) reported the concentrations of C-reactive protein (CRP), procalcitonin (PCT) and TREM-1 in the plasma of 76 patients in intensive care units. These markers were stronger in infected patients than in patients who did not present with SIRS. The plasma soluble TREM-1 levels had the greatest discriminative value, with an area under the receiver-operating characteristic curve of 0.97 , followed by procalcitonin (area under the curve $=0.85$ ) and C-reactive protein (area under the curve $=0.77$ ). At a cutoff level of $60 \mathrm{ng} / \mathrm{ml}$ (ELISA Human TREM-1 Quantikine ELISA Kit, R\&D Systems, Minneapolis, MN; sensitivity: $15.2 \mathrm{pg} / \mathrm{ml}$ ), soluble TREM-1 yielded a sensitivity of $96 \%$, a specificity of $89 \%$, a positive predictive value of $94 \%$ and a negative predictive value of $93 \%$ using StatView software (Abacus Concepts, Berkeley, CA) for 
Table 2. Qualitative and quantitative results for immunohistochemical staining for TREM 1 and ante-mortem s-TREM values: group 1.

\begin{tabular}{|c|c|c|c|c|c|}
\hline Case & Brain & Heart & Lung & Liver & Kidney \\
\hline 1 & $\begin{array}{l}\text { Quantitative } 0 ; \text { V } 4 \\
\text { Qualitative } 3\end{array}$ & $\begin{array}{l}\text { Quantitative 0; V } 3 \\
\text { Qualitative } 3\end{array}$ & $\begin{array}{l}\text { Quantitative macrophages 4(c); V } 4 \\
\text { Qualitative } 4\end{array}$ & $\begin{array}{l}\text { Quantitative hepatocytes } 3 \text { (c) } \\
\text { Bile ducts } 4 ; \text { V } 3 \\
\text { Qualitative } 3\end{array}$ & $\begin{array}{l}\text { Quantitative } \\
\text { glomeruli } 4 \text { tubules 3; V } 4 \\
\text { Qualitative } 3\end{array}$ \\
\hline 2 & $\begin{array}{l}\text { Quantitative } 0 ; \text { V } 1 \\
\text { Qualitative } 1\end{array}$ & $\begin{array}{l}\text { Quantitative 0; V } 2 \\
\text { Qualitative } 2\end{array}$ & $\begin{array}{l}\text { Quantitative macrophages 3(c); V } 2 \\
\text { Qualitative } 2\end{array}$ & $\begin{array}{l}\text { Quantitative hepatocytes } 2 \text { (c) } \\
\text { Bile ducts } 2 ; \text { V } 1 \\
\text { Qualitative } 2\end{array}$ & $\begin{array}{l}\text { Quantitative } \\
\text { glomeruli } 2 \text { tubules } 1 ; \mathrm{V} 2 \\
\text { Qualitative } 1\end{array}$ \\
\hline 3 & $\begin{array}{l}\text { Quantitative } 0 ; \text { V } 1 \\
\text { Qualitative } 1\end{array}$ & $\begin{array}{l}\text { Quantitative } 0 ; \text { V } 1 \\
\text { Qualitative } 1\end{array}$ & $\begin{array}{l}\text { Quantitative macrophages 1(c); V } 2 \\
\text { Qualitative } 2\end{array}$ & $\begin{array}{l}\text { Quantitative 0; V } 0 \\
\text { Qualitative } 0\end{array}$ & $\begin{array}{l}\text { Quantitative } \\
\text { glomeruli } 1 \text { tubules } 0 ; \mathrm{V} 1 \\
\text { Qualitative } 1\end{array}$ \\
\hline 4 & $\begin{array}{l}\text { Quantitative } 0 ; \vee 3 \\
\text { Qualitative } 3\end{array}$ & $\begin{array}{l}\text { Quantitative } 0 ; \text { V } 3 \\
\text { Qualitative } 4\end{array}$ & $\begin{array}{l}\text { Quantitative macrophages } 4 \text { (c); V } 3 \\
\text { Qualitative } 4\end{array}$ & $\begin{array}{l}\text { Quantitative hepatocytes } 2 \text { (c) } \\
\text { Bile ducts } 1 \text {; V } 2 \\
\text { Qualitative } 3\end{array}$ & $\begin{array}{l}\text { Quantitative } \\
\text { glomeruli } 3 \text { tubules 2; V } 3 \\
\text { Qualitative } 2\end{array}$ \\
\hline 5 & $\begin{array}{l}\text { Quantitative } 0 ; \vee 2 \\
\text { Qualitative } 1\end{array}$ & $\begin{array}{l}\text { Quantitative } 0 ; \mathrm{V} 1 \\
\text { Qualitative } 1\end{array}$ & $\begin{array}{l}\text { Quantitative macrophages } 1 \text { (c); V } 2 \\
\text { Qualitative } 1\end{array}$ & $\begin{array}{l}\text { Quantitative } 0 ; \vee 00 \\
\text { Qualitative } 0\end{array}$ & $\begin{array}{l}\text { Quantitative } \\
\text { glomeruli } 1 \text { tubules } 0 ; \mathrm{V} 1 \\
\text { Qualitative } 1\end{array}$ \\
\hline 6 & $\begin{array}{l}\text { Quantitative 0; V } 1 \\
\text { Qualitative } 2\end{array}$ & $\begin{array}{l}\text { Quantitative 0; V } 2 \\
\text { Qualitative } 1\end{array}$ & $\begin{array}{l}\text { Quantitative macrophages } 3 \text { (c); V } 2 \\
\text { Qualitative } 3\end{array}$ & $\begin{array}{l}\text { Quantitative hepatocytes } 1 \text { (c) } \\
\text { Bile ducts } 0 ; \text { V } 1 \\
\text { Qualitative } 2\end{array}$ & $\begin{array}{l}\text { Quantitative } \\
\text { Glomeruli } 2 \text { tubules 2; V } 2 \\
\text { Qualitative } 2\end{array}$ \\
\hline 7 & $\begin{array}{l}\text { Quantitative } 0 ; \vee 3 \\
\text { Qualitative } 2\end{array}$ & $\begin{array}{l}\text { Quantitative 0; V } 1 \\
\text { Qualitative } 2\end{array}$ & $\begin{array}{l}\text { Quantitative macrophages } 3 \text { (c); V } 3 \\
\text { Qualitative } 3\end{array}$ & $\begin{array}{l}\text { Quantitative hepatocytes } 1 \text { (c) } \\
\text { Bile ducts } 0 ; \text { V } 1 \\
\text { Qualitative } 2\end{array}$ & $\begin{array}{l}\text { Quantitative } \\
\text { glomeruli } 2 \text { tubules 2; V } 2 \\
\text { Qualitative } 2\end{array}$ \\
\hline 8 & $\begin{array}{l}\text { Quantitative } 0 ; \vee 3 \\
\text { Qualitative } 3\end{array}$ & $\begin{array}{l}\text { Quantitative 0; V } 2 \\
\text { Qualitative } 2\end{array}$ & $\begin{array}{l}\text { Quantitative macrophages } 3 \text { (c); V } 3 \\
\text { Qualitative } 3\end{array}$ & $\begin{array}{l}\text { Quantitative hepatocytes } 2 \text { (c) } \\
\text { Bile ducts 2; V } 2 \\
\text { Qualitative } 2\end{array}$ & $\begin{array}{l}\text { Quantitative } \\
\text { glomeruli } 2 \text { tubules 2; } \vee 3 \\
\text { Qualitative } 2\end{array}$ \\
\hline 9 & $\begin{array}{l}\text { Quantitative } 0 ; \vee 1 \\
\text { Qualitative } 1\end{array}$ & $\begin{array}{l}\text { Quantitative } 0 ; \mathrm{V} 2 \\
\text { Qualitative } 2\end{array}$ & $\begin{array}{l}\text { Quantitative macrophages 3(c); V } 2 \\
\text { Qualitative } 2\end{array}$ & $\begin{array}{l}\text { Quantitative hepatocytes } 2 \text { (c) } \\
\text { Bile ducts } 2 ; \text { V } 1 \\
\text { Qualitative } 2\end{array}$ & $\begin{array}{l}\text { Quantitative } \\
\text { glomeruli } 2 \text { tubules } 1 ; \mathrm{V} 2 \\
\text { Qualitative } 1\end{array}$ \\
\hline 10 & $\begin{array}{l}\text { Quantitative } 0 ; \text { V } 1 \\
\text { Qualitative } 1\end{array}$ & $\begin{array}{l}\text { Quantitative } 0 ; \mathrm{V} 1 \\
\text { Qualitative } 1\end{array}$ & $\begin{array}{l}\text { Quantitative macrophages 1(c); V } 2 \\
\text { Qualitative } 2\end{array}$ & $\begin{array}{l}\text { Quantitative } 0 ; \vee 0 \\
\text { Qualitative } 0\end{array}$ & $\begin{array}{l}\text { Quantitative } \\
\text { glomeruli } 1 \text { tubules } 0 ; \mathrm{V} 1 \\
\text { Qualitative } 1\end{array}$ \\
\hline 11 & $\begin{array}{l}\text { Quantitative } 0 ; \vee 3 \\
\text { Qualitative } 3\end{array}$ & $\begin{array}{l}\text { Quantitative } 0 ; \text { V } 3 \\
\text { Qualitative } 4\end{array}$ & $\begin{array}{l}\text { Quantitative macrophages } 4 \text { (c); V } 3 \\
\text { Qualitative } 4\end{array}$ & $\begin{array}{l}\text { Quantitative hepatocytes } 2 \text { (c) } \\
\text { Bile ducts } 1 \text {; V } 2 \\
\text { Qualitative } 3\end{array}$ & $\begin{array}{l}\text { Quantitative } \\
\text { glomeruli } 3 \text { tubules 2; V } 3 \\
\text { Qualitative } 2\end{array}$ \\
\hline 12 & $\begin{array}{l}\text { Quantitative 0; V } 2 \\
\text { Qualitative } 1\end{array}$ & $\begin{array}{l}\text { Quantitative } 0 ; \vee 1 \\
\text { Qualitative } 1\end{array}$ & $\begin{array}{l}\text { Quantitative macrophages } 1 \text { (c); V } 2 \\
\text { Qualitative } 1\end{array}$ & $\begin{array}{l}\text { Quantitative 0; V } 0 \\
\text { Qualitative } 0\end{array}$ & $\begin{array}{l}\text { Quantitative } \\
\text { glomeruli } 1 \text { tubules } 0 ; \mathrm{V} 1 \\
\text { Qualitative } 1\end{array}$ \\
\hline 13 & $\begin{array}{l}\text { Quantitative } 0 ; \text { V } 1 \\
\text { Qualitative } 2\end{array}$ & $\begin{array}{l}\text { Quantitative 0; V } 2 \\
\text { Qualitative } 1\end{array}$ & $\begin{array}{l}\text { Quantitative macrophages } 3 \text { (c); V } 2 \\
\text { Qualitative } 3\end{array}$ & $\begin{array}{l}\text { Quantitative hepatocytes } 1 \text { (c) } \\
\text { Bile ducts } 0 \text {; V } 1 \\
\text { Qualitative } 2\end{array}$ & $\begin{array}{l}\text { Quantitative } \\
\text { glomeruli } 2 \text { tubules 2; V } 2 \\
\text { Qualitative } 2\end{array}$ \\
\hline 14 & $\begin{array}{l}\text { Quantitative } 0 ; \vee 3 \\
\text { Qualitative } 2\end{array}$ & $\begin{array}{l}\text { Quantitative } 0 ; \text { V } 1 \\
\text { Qualitative } 2\end{array}$ & $\begin{array}{l}\text { Quantitative macrophages } 3 \text { (c); V } 3 \\
\text { Qualitative } 3\end{array}$ & $\begin{array}{l}\text { Quantitative hepatocytes } 1 \text { (c) } \\
\text { Bile ducts } 0 ; \text { V } 1 \\
\text { Qualitative } 2\end{array}$ & $\begin{array}{l}\text { Quantitative } \\
\text { gomeruli } 2 \text { tubules } 2 ; \text { V } 2 \\
\text { Qualitative } 2\end{array}$ \\
\hline
\end{tabular}

$\mathrm{V}$, intravascular staining; $n$, nuclear staining; $c$, cytoplasm staining. For quantitative analysis, the TREM-1 stained cells were counted and divided by the total number of cells and multiplied $\times 100$. Immunohistochemical positivity score was defined as: 0 , nuclear and/or cytoplasm staining absent; 1 , nuclear and/or cytoplasmic staining, 25\%; 2, nuclear and/or cytoplasmic staining, 50\%; 3, nuclear and/or cytoplasmic staining, 75\%; 4, nuclear and/or cytoplasm staining, $100 \%$. Immunohistochemical staining in blood vessel was quantified as: 0 , absent; 1 , low, 25\%; 2, sufficient, 50\%; 3, good, 75\%; 4, considerable, 100\%. Five degrees of staining intensity were defined as: 0 , absent; 1 , low, $25 \% ; 2$, sufficient, $50 \% ; 3$, good, $75 \%$; , considerable.

the statistical analysis; a 2 -tailed $p$ value $\leq 0.05$ was considered statistically significant). Rivera-Chavez and Minei (2009) reported an s-TREM-1 cut-off value of $230 \mathrm{pg} / \mathrm{ml}$, which yielded a sensitivity of $98 \%$ and specificity of $91 \%$ for differentiating patients with SIRS from those with infection. Patients with infection had significantly higher s-TREM-1 concentrations than patients with SIRS (median $398 \mathrm{pg} / \mathrm{ml}$ vs. $78 \mathrm{pg} / \mathrm{ml}$, respectively).

Wang and Chen (2011) reported findings concerning 32 septic patients and 24 patients affected by SIRS in intensive care units. Compared to the SIRS group, s-TREM-1 was significantly increased in the sepsis group. In addition to its use as a diagnostic biomarker, s-TREM-1 concentration can used to determine the prognosis of septic patients. Gibot et al. (2005) measured plasma s-TREM-1 concentration together with monocyte expression of TREM-1 in 63 septic patients to determine prognosis.

Promising results with s-TREM-1 as diagnostic sepsis marker have been reported. Brenner et al. (2016) reported that the plasma level of s-TREM-1 was a better biomarker for identifying patients with septic shock than the most commonly used acute phase proteins, CRP and PCT.

Gibot et al. (2007) reported that measurement of serum PCT together with s-TREM-1 concentrations could be useful for detecting nosocomial sepsis. Aksaray et al. (2016) also reported that s-TREM-1 and PCT are excellent markers for early diagnosis of sepsis. Although further studies are required to confirm them, our preliminary findings indicate that s-TREM-1 could enable postmortem diagnosis of sepsis with good sensitivity and specificity. 
Table 3. Qualitative and quantitative results for immunohistochemical staining for TREM 1: group 2.

\begin{tabular}{|c|c|c|c|c|c|}
\hline CASE & Brain & Heart & Lung & Liver & Kidney \\
\hline Case 1 & $\begin{array}{l}\text { Quantitative 0; V } 2 \\
\text { Qualitative } 0\end{array}$ & $\begin{array}{l}\text { Quantitative 0; V } 0 \\
\text { Qualitative } 0\end{array}$ & $\begin{array}{l}\text { Quantitative macrophages } 1(\mathrm{c}) ; \vee 2 \\
\text { Qualitative } 1\end{array}$ & $\begin{array}{l}\text { Quantitative 0; V } 0 \\
\text { Qualitative } 0\end{array}$ & $\begin{array}{l}\text { Quantitative } \\
\text { Glomeruli } 0 \text { tubules } 1 ; \vee 00 \\
\text { Qualitative } 1\end{array}$ \\
\hline Case 2 & $\begin{array}{l}\text { Quantitative } 0 ; \vee 22 \\
\text { Qualitative } 2\end{array}$ & $\begin{array}{l}\text { Quantitative } 0 ; \text { V } 2 \\
\text { Qualitative } 2\end{array}$ & $\begin{array}{l}\text { Quantitative macrophages 2(c); V } 2 \\
\text { Qualitative } 2\end{array}$ & $\begin{array}{l}\text { Quantitative hepatocytes } 1 \text { (c) } \\
\text { Bile ducts } 1 ; \vee 1 \\
\text { Qualitative } 1\end{array}$ & $\begin{array}{l}\text { Quantitative } \\
\text { Glomeruli } 2 \text { tubules 2; V } 2 \\
\text { Qualitative } 1\end{array}$ \\
\hline Case 3 & $\begin{array}{l}\text { Quantitative 0; V } 1 \\
\text { Qualitative } 0\end{array}$ & $\begin{array}{l}\text { Quantitative } 0 ; \text { V } 1 \\
\text { Qualitative } 1\end{array}$ & $\begin{array}{l}\text { Quantitative } 0(\mathrm{c}) \text {; V } 1 \\
\text { Qualitative } 1\end{array}$ & $\begin{array}{l}\text { Quantitative 0; V } 0 \\
\text { Qualitative } 0\end{array}$ & $\begin{array}{l}\text { Quantitative } \\
\text { Glomeruli } 0 \text { tubules } 1 ; \vee 00 \\
\text { Qualitative } 1\end{array}$ \\
\hline Case 4 & $\begin{array}{l}\text { Quantitative 0; V } 1 \\
\text { Qualitative } 2\end{array}$ & $\begin{array}{l}\text { Quantitative 0; V } 1 \\
\text { Qualitative } 2\end{array}$ & $\begin{array}{l}\text { Quantitative } 0 \text { (c); V } 2 \\
\text { Qualitative } 1\end{array}$ & $\begin{array}{l}\text { Quantitative hepatocytes } 1 \text { (c) } \\
\text { Bile ducts } 0 \text {; V } 1 \\
\text { Qualitative } 2\end{array}$ & $\begin{array}{l}\text { Quantitative } \\
\text { Glomeruli } 2 \text { tubules } 0 ; \mathrm{V} 1 \\
\text { Qualitative } 2\end{array}$ \\
\hline Case 5 & $\begin{array}{l}\text { Quantitative 0; V } 4 \\
\text { Qualitative } 3\end{array}$ & $\begin{array}{l}\text { Quantitative } 0 ; \text { V } 2 \\
\text { Qualitative } 3\end{array}$ & $\begin{array}{l}\text { Quantitative macrophages } 3 \text { (c); } \vee 3 \\
\text { Qualitative } 2\end{array}$ & $\begin{array}{l}\text { Quantitative hepatocytes } 2 \text { (c) } \\
\text { Bile ducts } 1 \text {; V } 2 \\
\text { Qualitative } 2\end{array}$ & $\begin{array}{l}\text { Quantitative } \\
\text { Glomeruli } 2 \text { tubules } 3 ; \mathrm{V} 3 \\
\text { Qualitative } 2\end{array}$ \\
\hline Case 6 & $\begin{array}{l}\text { Quantitative } 0 ; \vee 22 \\
\text { Qualitative } 2\end{array}$ & $\begin{array}{l}\text { Quantitative 0; V } 1 \\
\text { Qualitative } 2\end{array}$ & $\begin{array}{l}\text { Quantitative macrophages } 2 \text { (c); } \vee 2 \\
\text { Qualitative } 2\end{array}$ & $\begin{array}{l}\text { Quantitative hepatocytes } 0 \text { (c) } \\
\text { Bile ducts } 1 \text {; V } 0 \\
\text { Qualitative } 1\end{array}$ & $\begin{array}{l}\text { Quantitative } \\
\text { Glomeruli } 1 \text { tubules } 0 ; \mathrm{V} 1 \\
\text { Qualitative } 1\end{array}$ \\
\hline Case 7 & $\begin{array}{l}\text { Quantitative } 0 ; \vee 3 \\
\text { Qualitative } 3\end{array}$ & $\begin{array}{l}\text { Quantitative 0; V } 3 \\
\text { Qualitative } 2\end{array}$ & $\begin{array}{l}\text { Quantitative macrophages } 4 \text { (c); V } 4 \\
\text { Qualitative } 3\end{array}$ & $\begin{array}{l}\text { Quantitative hepatocytes } 3 \text { (c) } \\
\text { Bile ducts } 2 ; \text { V } 2 \\
\text { Qualitative } 3\end{array}$ & $\begin{array}{l}\text { Quantitative } \\
\text { Glomeruli } 4 \text { tubules 3; v } 2 \\
\text { Qualitative } 3\end{array}$ \\
\hline Case 8 & $\begin{array}{l}\text { Quantitative } 0 ; \text { V } 1 \\
\text { Qualitative } 2\end{array}$ & $\begin{array}{l}\text { Quantitative } 0 ; \text { V } 2 \\
\text { Qualitative } 1\end{array}$ & $\begin{array}{l}\text { Quantitative macrophages } 1 \text { (c); } \vee 2 \\
\text { Qualitative } 2\end{array}$ & $\begin{array}{l}\text { Quantitative hepatocytes } 1 \text { (c) } \\
\text { Bile ducts } 0 ; \text { V } 0 \\
\text { Qualitative } 1\end{array}$ & $\begin{array}{l}\text { Quantitative } \\
\text { Glomeruli } 2 \text { tubules 2; V } 2 \\
\text { Qualitative } 1\end{array}$ \\
\hline Case 9 & $\begin{array}{l}\text { Quantitative } 0 ; \vee 2 \\
\text { Qualitative } 2\end{array}$ & $\begin{array}{l}\text { Quantitative } 0 ; \vee 2 \\
\text { Qualitative } 2\end{array}$ & $\begin{array}{l}\text { Quantitative macrophages 2(c); V } 2 \\
\text { Qualitative } 2\end{array}$ & $\begin{array}{l}\text { Quantitative hepatocytes } 1 \text { (c) } \\
\text { Bile ducts } 1 ; \mathrm{V} 1 \\
\text { Qualitative } 1\end{array}$ & $\begin{array}{l}\text { Quantitative } \\
\text { Glomeruli } 2 \text { tubules 2; V } 2 \\
\text { Qualitative } 1\end{array}$ \\
\hline Case 10 & $\begin{array}{l}\text { Quantitative 0; V } 1 \\
\text { Qualitative } 0\end{array}$ & $\begin{array}{l}\text { Quantitative } 0 ; \text { V } 1 \\
\text { Qualitative } 1\end{array}$ & $\begin{array}{l}\text { Quantitative } 0 \text { (c); V } 1 \\
\text { Qualitative } 1\end{array}$ & $\begin{array}{l}\text { Quantitative } 0 ; \vee 00 \\
\text { Qualitative } 0\end{array}$ & $\begin{array}{l}\text { Quantitative } \\
\text { Glomeruli } 0 \text { tubules } 1 ; \mathrm{V} 0 \\
\text { Qualitative } 1\end{array}$ \\
\hline Case 11 & $\begin{array}{l}\text { Quantitative 0; V } 1 \\
\text { Qualitative } 2\end{array}$ & $\begin{array}{l}\text { Quantitative 0; V } 1 \\
\text { Qualitative } 2\end{array}$ & $\begin{array}{l}\text { Quantitative } 0 \text { (c); V } 2 \\
\text { Qualitative } 1\end{array}$ & $\begin{array}{l}\text { Quantitative hepatocytes } 1 \text { (c) } \\
\text { Bile ducts } 0 ; \text { V } 1 \\
\text { Qualitative } 2\end{array}$ & $\begin{array}{l}\text { Quantitative } \\
\text { Glomeruli } 2 \text { tubules } 0 ; \mathrm{V} 1 \\
\text { Qualitative } 2\end{array}$ \\
\hline Case 12 & $\begin{array}{l}\text { Quantitative 0; V } 4 \\
\text { Qualitative } 3\end{array}$ & $\begin{array}{l}\text { Quantitative 0; V } 2 \\
\text { Qualitative } 3\end{array}$ & $\begin{array}{l}\text { Quantitative macrophages } 3 \text { (c); V } 3 \\
\text { Qualitative } 2\end{array}$ & $\begin{array}{l}\text { Quantitative hepatocytes } 2 \text { (c) } \\
\text { Bile ducts } 1 \text {; V } 2 \\
\text { Qualitative } 2\end{array}$ & $\begin{array}{l}\text { Quantitative } \\
\text { Glomeruli } 2 \text { tubules } 3 ; \text { V } 3 \\
\text { Qualitative } 2\end{array}$ \\
\hline Case 13 & $\begin{array}{l}\text { Quantitative 0; V } 2 \\
\text { Qualitative } 2\end{array}$ & $\begin{array}{l}\text { Quantitative 0; V } 1 \\
\text { Qualitative } 2\end{array}$ & $\begin{array}{l}\text { Quantitative macrophages } 2 \text { (c); V } 2 \\
\text { Qualitative } 2\end{array}$ & $\begin{array}{l}\text { Quantitative hepatocytes } 0 \text { (c) } \\
\text { Bile ducts } 1 \text {; V } 0 \\
\text { Qualitative } 1\end{array}$ & $\begin{array}{l}\text { Quantitative } \\
\text { Glomeruli } 1 \text { tubules } 0 ; \mathrm{V} 1 \\
\text { Qualitative } 1\end{array}$ \\
\hline Case 14 & $\begin{array}{l}\text { Quantitative } 0 ; \text { V } 3 \\
\text { Qualitative } 3\end{array}$ & $\begin{array}{l}\text { Quantitative } 0 ; \text { V } 3 \\
\text { Qualitative } 2\end{array}$ & $\begin{array}{l}\text { Quantitative macrophages } 4 \text { (c); V } 4 \\
\text { Qualitative } 3\end{array}$ & $\begin{array}{l}\text { Quantitative hepatocytes } 3 \text { (c) } \\
\text { Bile ducts } 2 ; \text { V } 2 \\
\text { Qualitative } 3\end{array}$ & $\begin{array}{l}\text { Quantitative } \\
\text { Glomeruli } 4 \text { tubules 3; V } 2 \\
\text { Qualitative } 3\end{array}$ \\
\hline
\end{tabular}

$\mathrm{V}$, intravascular staining; $\mathrm{n}$, nuclear staining; $c$, cytoplasmic staining. For quantitative analysis, the TREM-1 stained cells were counted and divided by the total number of cells and multiplied $\times 100$. Immunohistochemical positivity score was defined as: 0 , nuclear and/or cytoplasm staining absent; 1 , nuclear and/or cytoplasmic staining, 25\%; 2, nuclear and/or cytoplasmic staining, 50\%; 3, nuclear and/or cytoplasmic staining, 75\%; 4, nuclear and/or cytoplasm staining, $100 \%$. Immunohistochemical staining in blood vessel was quantified as: 0, absent; 1, low, 25\%; 2, sufficient, 50\%; 3, good, 75\%; 4, considerable, 100\%. Five degrees of staining intensity were defined as: 0 , absent; 1, low, 25\%; 2, sufficient, $50 \%$; 3, good, 75\%; 4, considerable.

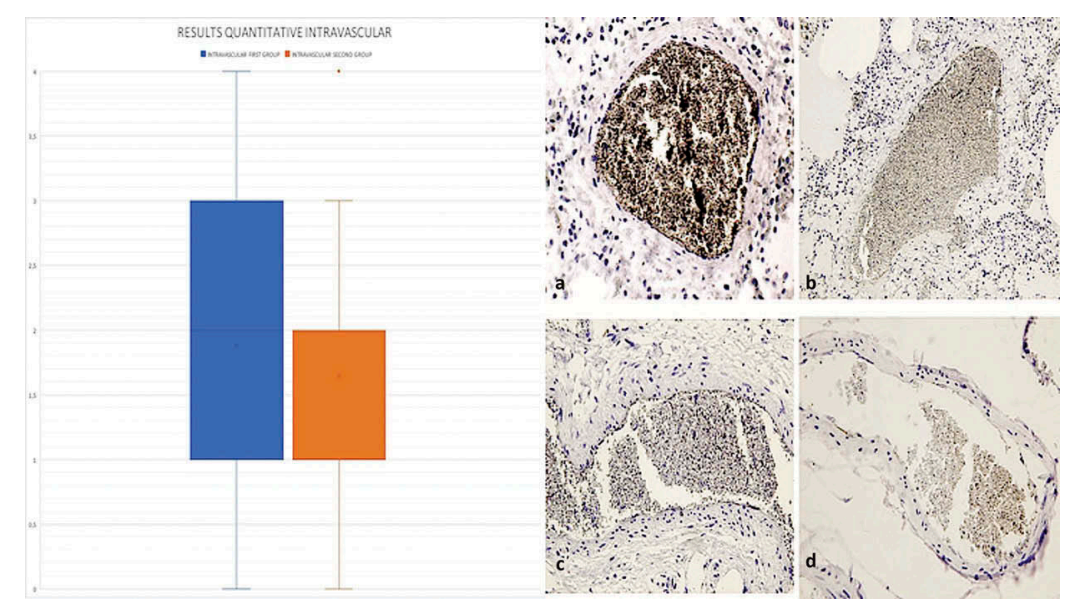

Figure 1. Box and whiskers plot of intravascular immunostaining by anti-TREM-1. Staining of the TREM-1 antibody in blood vessels of kidney a) x 200, lung b) x 100, myocardium c) x 200, and brain d) x 200. 




Figure 2. a) TREM-1 staining (arrows) in cytoplasm of the myelomonocyte line. $x$ 60. b) Weak TREM-1 staining (arrow) in hepatocytes. x 200 .

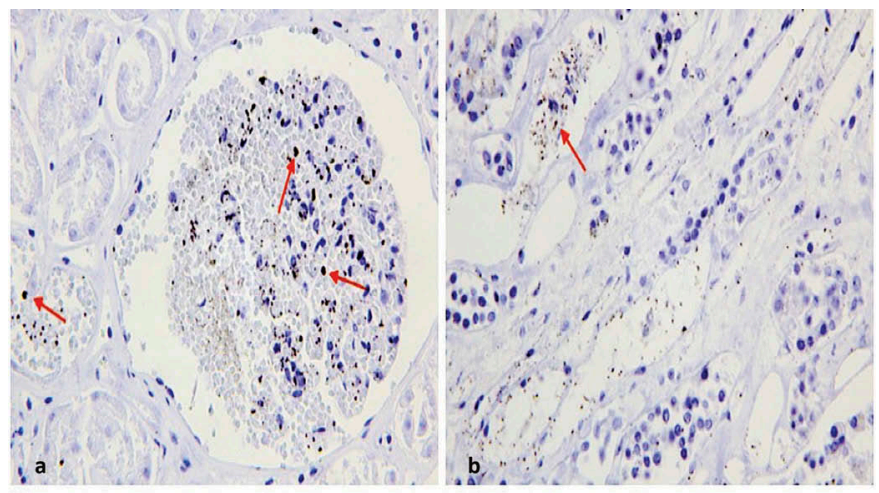

Figure 3. TREM-1 staining (arrows) in glomeruli a) x 100 and renal tubules (arrows) b) x 200 .

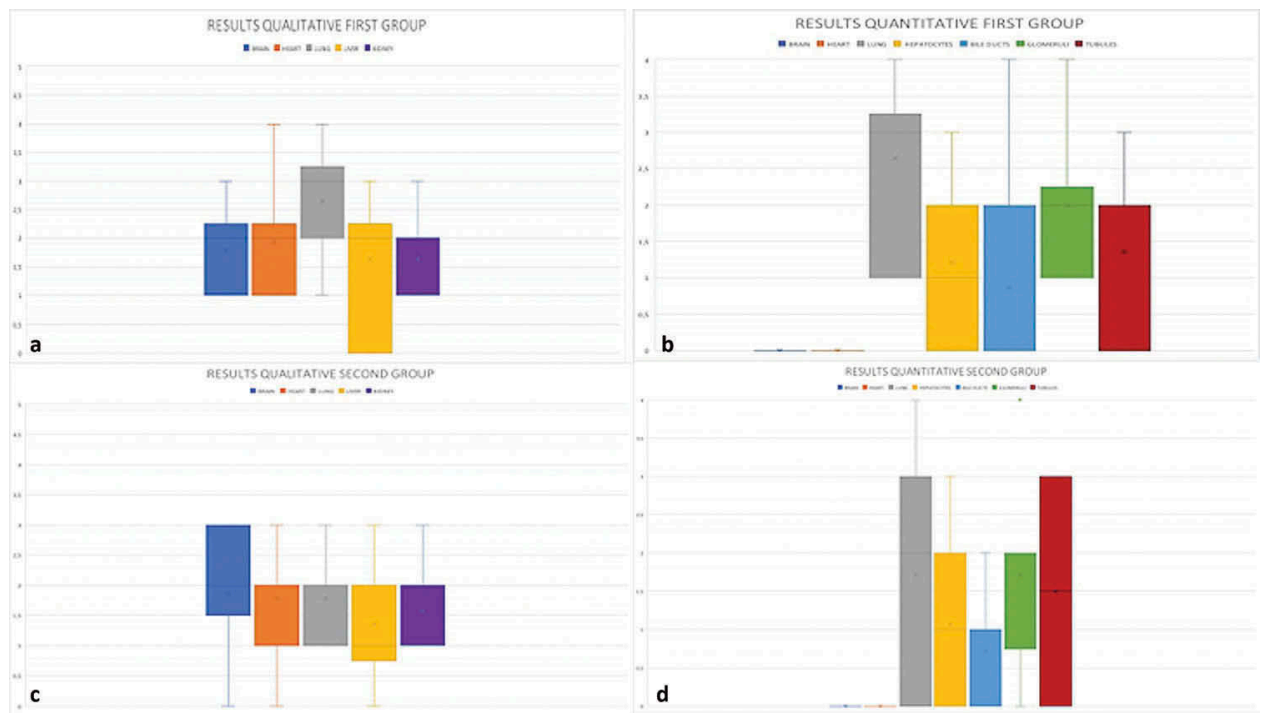

Figure 4. Box and whiskers plot showing greater immunohistochemical reaction, both quantitatively and qualitatively, in the group with documented ante-mortem s-TREM-1. 
Our findings for lung samples suggest that s-TREM-1 might be produced principally in the cells of the monocyte line. Our findings for liver and kidney samples could be related to the pathway of excretion of TREM-1. TREM-1 staining in kidney tubules, hepatocytes and liver ducts are a normal physiological finding related to the elimination of this protein from the circulation.

One limitations of our study is our use of a relatively small number of subjects. Precise criteria, however, were used for selecting subjects. Also, detailed immunohistochemical studies TREM-1 are not available, which precluded comparison of our observations with others.

We believe that our measurement of s-TREM-1 may be promising for making postmortem diagnoses of sepsis. Our immunohistochemical technique currently is more convenient compared to searching for s-TREM-1 in serum or other biological fluids (Bellos et al. 2018). It is important to compare the diagnostic accuracy of s-TREM-1 with other biomarkers, such as CRP and PCT. the variation in the threshold values used (between 77.5 and $1707.35 \mathrm{pg} / \mathrm{ml}$ ), however, limit the value of these findings in clinical practice (Bellos et al. 2018). Also TREM-1 could be stained in patients with non-infective inflammatory diseases such as psoriasis, ulcerative colitis or immune complex vasculitis. Moreover, TREM-1 could be stained in patients affected by hemorrhagic shock, reperfusion injury or by a peptic ulcer caused by H. pylori (Gibot et al. 2008; Yasuda et al. 2008; Barbatzas and Pimentel 2011).

If s-TREM-1 staining can be found in cases un related to sepsis, the anti-TREM-1 antibody could be combined with an anti-procalcitonin and/or lactoferrin antibody to produce considerable sensitivity and specificity in sepsis related cases of death (Maiese et al. 2017; Galassi et al. 2018). Immunohistochemical staining using the antiTREM-1 antibody can be a useful method for postmortem diagnosis of sepsis (Palmiere et al. 2013).

\section{Disclosure statement}

No potential conflict of interest was reported by the authors.

\section{References}

Aksaray S, Alagoz P, Inan A, Cevan S, Ozgultekin A. 2016. Diagnostic value of sTREM-1 and procalcitonin levels in the early diagnosis of sepsis. North Clin Istanb. 3:175-182. Arízaga-Ballesteros V, Alcorta-Garcíaa MR, Lázaro-Martínez LC, Amézquita-Gómez JM, Alanís-Cajero JM, Villela L, Castorena-Torres F, Lara-Díaz VJ. 2015. Can s-TREM-1 predict septic shock \& death in late-onset neonatal sepsis? A pilot study. Int J Infect Dis. 30:27-32.
Barbatzas C, Pimentel M. 2011. Serum s-TREM-1 as a surrogate marker of treatment outcome in patients with peptic ulcer disease. Dig Dis Sci. 56:3590-3595.

Barraud D, Gibot S. 2011. Triggering receptor expressed on myeloid cell-1. Crit Care Clin. 27:265-279.

Bellos I, Fitrou G, Daskalakis G, Thomakos N, Papantoniou N, Pergialiotis V. 2018. Soluble TREM-1 as a predictive factor of neonatal sepsis: a meta-analysis. Inflam Res. 67:571-578.

Bleharski JR, Kiessler V, Buonsanti C, Sieling PA, Stenger S, Colonna M, Modlin RL. 2003. A role for triggering receptor expressed on myeloid cells- 1 in host defense during the early-induced and adaptive phases of the immune response. J Immunol. 170:3812-3818.

Bouchon A, Dietrich J, Colonna M. 2000. Cutting edge: inflammatory responses can be triggered by TREM-1, a novel receptor expressed on neutrophils and monocytes. J Immunol. 164:4991-4995.

Bouchon A, Facchetti F, Weigand MA, Colonna M. 2001. TREM-1 amplifies inflammation and is a crucial mediator of septic shock. Nature. 410:1103-1107.

Brenner T, Uhle F, Fleming T. 2016. Soluble TREM-1 as a diagnostic and prognostic biomarker in patients with septic shock-an observational clinical study. Biomarkers. 20:1-23.

Carsin H, Assicot M, Feger F, Roy O, Pennacino I, Le Bever H, Ainaud P, Bohuon C. 1997. Evolution and significance of circulating procalcitonins levels compared with IL-6, $\mathrm{TNFa}$ and endotoxin levels early after thermal injury. Burns. 23:218-224.

Fischer AH, Jacobson KA, Rose J, Zeller R. 2008. Hematoxylin and eosin staining of tissue and cell sections. CSH Protoc. doi:10.1101/pdb.prot4986

Galassi A, Turatello L, De Salvia A, Neri M, Turillazzi E, La Russa R, Viola RV, Frati P, Fineschi V. 2018. Septic cardiomyopathy: the value of lactoferrin and CD15 as specific markers to corroborate a definitive diagnosis. Int J Immunopathol Pharmacol. 32:2058738418776526.

Giamarellos-Bourboulis EJ, Zakynthinos S, Baziaka F, Papadomichelakis E, Virtzili S, Koutoukas P, Armaganidis A, Giamarellou H, Roussos C. 2006. Soluble triggering receptor expressed on myeloid cells 1 as an anti-inflammatory mediator in sepsis. Intens Care Med. 32:237-243.

Gibot S, Cravoisy A, Dupays R, Gibot S, Cravoisy A, Dupays R. 2007. Combined measurement of procalcitonin and soluble TREM-1 in the diagnosis of nosocomial sepsis. Scand J Infect Dis. 36:604-608.

Gibot S, Cravoisy A, Kolopp-Sarda MN, Béné MC, Faure G, Bollaert PE, Levy B. 2005. Time-course of sTREM (soluble triggering receptor expressed on myeloid cells)-1, procalcitonin, and C-reactive protein plasma concentrations during sepsis. Crit Care Med. 33:792-796.

Gibot S, Kolopp-Sarda MN, Béné MC, Cravoisy A, Levy B, Faure GC, Bollaert PE. 2004. Plasma level of a triggering receptor expressed on myeloid cells-1: its diagnostic accuracy in patients with suspected sepsis. Ann Int Med. 141:9-15.

Gibot S, Massin F, Alauzet C, Montemont C, Lozniewski A, Bollaert PE, Levy B. 2008. Effects of the TREM-1 pathway modulation during mesenteric ischemia-reperfusion in rats. Crit Care Med. 36:504-510.

Herwig MC, Tsokos M, Hermanns MI, Kirkpatrick CJ, Müller AM. 2013. Vascular endothelial Cadherin expression in lung specimens of patients with sepsis-induced 
acute respiratory distress syndrome and endothelial cell cultures. Pathobiology. 80:245-251.

Latour-Pérez J, Alcalá-López A, García-García MA, SánchezHernández JF, Abad-Terrado C, Viedma-Contreras JA, Masiá-Canuto M, Broch-Porcar MJ, Arizo-León D, González-Tejera M, Bonilla-Rovira F, Gutiérrez F. 2010. Prognostic value of the s-TREM-1 plasma values in patients with sepsis: a cohort study. Clin Biochem. 43:720-724.

Maiese A, Del Nonno F, Dell'Aquila M, Moauro M, Baiocchini A, Mastracchio A, Bolino G. 2017. Postmortem diagnosis of sepsis: a preliminary immunohistochemical study with an anti-procalcitonin antibody. Leg Med (Tokyo). 28:1-5.

Müller AM, Gruhn KM, Herwig MC, Tsokos M. 2008. VEcadherin and ACE: markers for sepsis in post mortem examination? Leg Med. 10:257-263.

Oku R, Oda S, Nakada TA, Sadahiro T, Nakamura M, Hirayama Y, Abe R, Tateishi Y, Ito M, Iseki T, Hirasawa H. 2013. Differential pattern of cell-surface and soluble TREM-1 between sepsis and SIRS. Cytokine. 61:112-117.

Palmiere C, Bardy D, Mangin P, Augsburger M. 2013. Value of s-TREM-1, procalcitonin and CRP as laboratory parameters for postmortem diagnosis of sepsis. J Infect. 67:545-555.

Rivera-Chavez FA, Minei JP. 2009. Soluble triggering receptor expressed on myeloid cells- 1 is an early marker of infection in the surgical intensive care unit. Surg Infect. 10:435-439.

Singer M, Deutschman CS, Seymour CW, Shankar-Hari M, Annane D, Bauer M, Bellomo R, Bernard GR, Chiche JD, Coopersmith CM, Hotchkiss RS, Levy MM, Marshall JC, Martin GS, Opal SM, Rubenfeld GD, van der Poll T, Vincent JL, Angus DC. 2016. The third international consensus definitions for sepsis and septic shock (sepsis3). J Am Med Assoc. 315:801-810.

Tsokos M. 2007. Postmortem diagnosis of sepsis. For Sci Int. 165:155-164.

Wang H, Chen B. 2011. Diagnostic role of soluble triggering receptor expressed on myeloid cell-1 in patients with sepsis. World J Emerg Med. 2:190-194.

Weiss G, Lai C, Fife ME, Grabiec AM, Tildy B, Snelgrove RJ, Xin G, Lloyd CM, Hussell T. 2017. Reversal of TREM-1 ectodomain shedding and improved bacterial clearance by intranasal metalloproteinase inhibitors. Mucosal Immunol. 10:1021-1030.

Wu Y, Wang F, Fan X, Bao R, Bo L, Li J, Deng X. 2012. Accuracy of plasma s-TREM-1 for sepsis diagnosis in systemic inflammatory patients: a systematic review and meta-analysis. Crit Care. 16:R229.

Yasuda T, Takeyama Y, Ueda T, Shinzeki M, Sawa H, Takahiro N, Kamei K, Ku Y, Kuroda Y. 2008. Increased levels of soluble triggering receptor expressed on myeloid cells- 1 in patients with acute pancreatitis. Crit Care Med. 36:2048-2053.

Yuan Z, Mehta HJ, Mohammed K, Nasreen N, Roman R, Brantly M, Sadikot RT. 2014. TREM-1 is induced in tumor associated macrophages by cyclo-oxygenase pathway in human non-small cell lung cancer. PLoS One. 19: e94241.

Yuan Z, Syed MA, Panchal D, Yuan Z, Syed MA, Panchal D, Rogers D, Joo M, Sadikot RT. 2012. Curcumin mediated epigenetic modulation inhibits TREM-1 expression in response to lipopolysaccharide. Int J Biochem Cell Biol. 44:2032-2043. 